\title{
Sensible label-free bio-sensing: Towards in situ biopsy
}

Hirak Kumar Patra

The self-archived postprint version of this conference paper is available at Linköping University Institutional Repository (DiVA):

http://urn.kb.se/resolve?urn=urn:nbn:se:liu:diva-158601

N.B.: When citing this work, cite the original publication.

Patra, H. K., (2018), Sensible label-free bio-sensing: Towards in situ biopsy, 2018 IEEE SENSORS, 1721-1724. https://doi.org/10.1109/ICSENS.2018.8589723

Original publication available at:

https://doi.org/10.1109/ICSENS.2018.8589723

Copyright: Institute of Electrical and Electronics Engineers

http://www.ieee.org/index.html

(C)2018 IEEE. Personal use of this material is permitted. However, permission to reprint/republish this material for advertising or promotional purposes or for creating new collective works for resale or redistribution to servers or lists, or to reuse any copyrighted component of this work in other works must be obtained from the IEEE. 


\title{
Sensible label-free bio-sensing: Towards in situ biopsy
}

\author{
Hirak K Patra ${ }^{1,2,3^{*}}$ \\ ${ }^{1}$ Department of Chemical Engineering and Biotechnology, University of Cambridge, Cambridge, UK \\ ${ }^{2}$ Wolfson College, University of Cambridge, Cambridge, UK \\ 3Department of Clinical and Experimental Medicine (IKE), Linkoping University, Linkoping, Sweden \\ *hp401@cam.ac.uk
}

\begin{abstract}
Integrative in situ bio-sensing approaches became ominous to advance early stage detection of complex and heterogeneous disease like cancer, chronic lung, inflammation, coronary syndromes etc. Particularly in cancer, we can save million lives with existing therapeutic strategies if we can detect them at their very early stage. Unfortunately, cancer offers very rare distinctly identifiable cues during its onset to identify them and posing the technological challenges to spot them at their very early stage. We have developed an aptamer based integrated switchable rare event sensing platform using variable angle spectroscopic ellipsometry to spot in situ, readout and monitor rare events in real-time in complex heterogeneous cell population. The developed aptasensors is being tested with spotting prostate cancer cell PC3 in a blood mimicking population of human peripheral blood mononuclear cells (PBMC). The proof-of-concept data showed that the developed platform can detect in situ up to 10 target cells/ml with $10^{9}$ background cells. The integrated sensor is designed with switchable surface to safely capture and release the target cells for further clinical validation and analysis for advanced diagnosis. We envision this alternate integrated bio-sensors strategy will be an important step towards the development of label-free in situ biopsy technique for rare event detection and early stage detection of cancer.
\end{abstract}

Keywords-Label-free, Whole cell Biosensor; Aptasensor; on/off switchable surface; early stage detection; Ellipsometry

\section{INTRODUCTION}

Detection of early rare events in human can help to diagnose fatal diseases including cancer and can save thousands of lives [1]. However, only very few limited cues are available during early onset of such disease. On top of that these cues and biomarkers are not often uniquely distinct to be spotted clearly by sensing devices. The heterogeneous contributions to the similar biomarker due to several altered physiological condition makes it more difficult to detect [2]. Despite of these challenges, there are number of advanced biomedical methods have been developed those can precisely detect molecules even at the pico molar range[3].

Unfortunately, disease like cancer, inflammation etc. are not only heterogeneous but also dynamic in nature $[4,5]$. They keep changing their expression molecule before being detected by our normal natural immune systems and leave us with this reality that there is no unique biomarker exist even at present time for the specific detection of cancer [6]. Therefore, proteomic, transcriptomic, lipidomic and metabolomics molecular detection systems and high throughput multiplex screening system are emerging as the validation tool for confirmatory diagnosis[7]. Despite these confirmatory support for clinical validation, none of them are easy accessible and suitable to design for an early stage spotting set up. Aptamer is rapidly emerging as a sensing molecule for their selective target recognition[8]. Furthermore, rare cell-selective aptamers can be achieved without targeting exact molecule on the cell surface. Systematic evolution of ligands by exponential enrichment (SELEX) offers the opportunity to select a whole cell and it could be possible that the derived aptamers could recognise several entity simultaneously on the target cell surface and that unique combination might be absent in similar cells[9-11]. There are number of aptamers are developing that could potentially bind to their unique targets. However, sensing through aptamer is not enough for their translational applications into the real clinical system until they are crossevaluated with the molecular diagnostics with standard operating protocols (SOP).

In the present approach, we are proposing a novel in situ label free real time switchable bio-sensing platform that could potentially exploit to develop dynamic physiological event for detection of rare events such as early stage detection of cancer (in situ biopsies).

\section{EXPERIMENTAL}

\section{A. Design of the sensor}

The present working hypothesis is based on the ellipsometry spectroscopy for label free sensing of the structural aspects of aptamer-cancer cell interactions[12]. The basic principle has been tested with cancer specific aptamer immobilized gold surface as described in Figure 1. In brief, preceding to immobilisation of wy-5a aptamer (5'TGCCACTACAGCTGGTTCGGTTTGGTGACTTCGTTCTT CGTTGTGGTGCTTAGTGGC-3') specific to PC3 cell (target cell), the gold coated silicon wafer surface was prudently washed with appropriate solvent sequentially (hexane-acetoneethanol-MilliQ). A uniform self-assembled monolayer of 3mercaptopropionic acid (MPA) was developed by incubating $50 \mathrm{mM}$ MPA for $24 \mathrm{~h}$. The MPA-modified sensor surface was then washed several times with Milli-Q water and completely removed trace amount of unreacted MPA. The functionalised sensor surface were activated using $20 \mathrm{mM}$ EDC and $30 \mathrm{mM}$ sulfo-NHS solutions in MES for $2 \mathrm{~h}$. We have co-grafted the amine functionalised $5^{\prime} \mathrm{NH}_{2}-\left(\mathrm{CH}_{2}\right)_{6}-\mathrm{Wy}-5 \mathrm{a}$ aptamer and amine terminated Poly ( $\mathrm{N}$-isopropylacrylamide) (PNIPAAM)at 
the same time and the ratio was adjusted for optimal binding

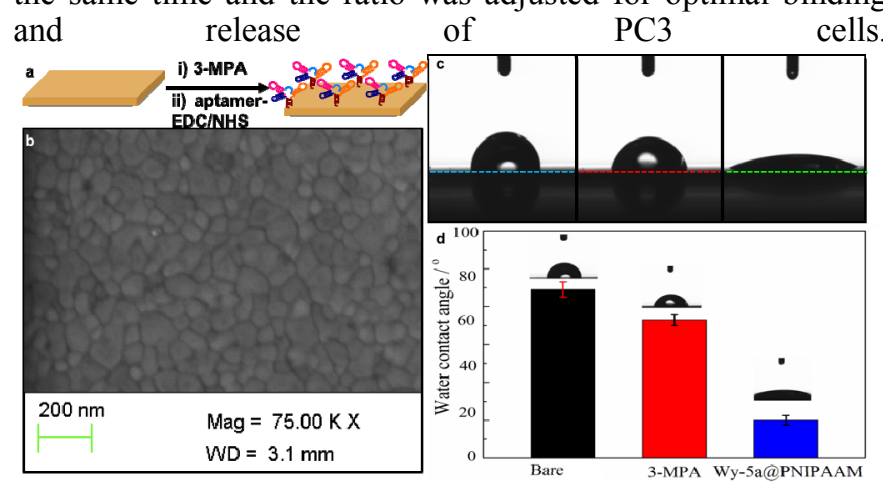

Figure 1: Fabrication, basic schematic description and surface properties of the platform.

\section{B. Characterisation the sensor}

$V A S E$ : To optimise the sensor surface, we have used Variable angle Spectroscopic Ellipsometer (VASE from J.A. Woollam Co., Inc.) that can provide precise information from the immobilized sensor layer. The design described in Figure 2 has been used for in situ measurements using a variable angle spectroscopic ellipsometry (VASE) at an optimised angle of incidence of $68^{\circ}$ in the spectral range $350-1050 \mathrm{~nm}$ to get an incident wavelength independent measure[13]. A specially designed cuvette (Figure 2c) have been used for the optical readout from the sensor surface[13] along with a magnetic stirrer and a flow system for simulating the blood flow. The sensor was first incubated in PBS at $\mathrm{pH} 7.4$ and performed the spectral measurements without target cells. Followed by target cells have been added and the corresponding spectral changes monitored. A negative control has been kept for each individual set of experiment.

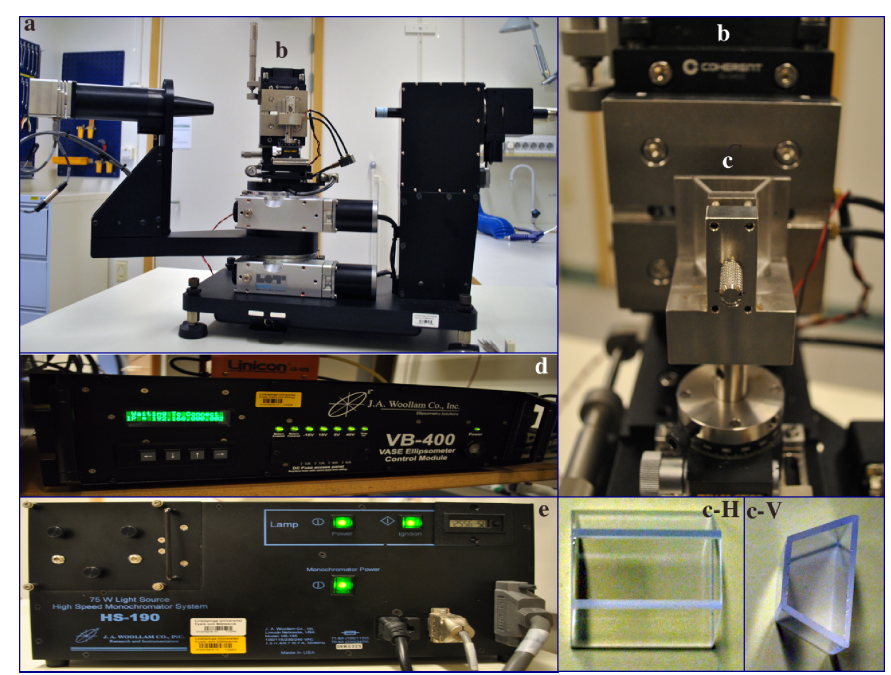

Figure 2: Basic set up (a) and principal components (b-c) of the VASE and real time monitoring (d-e)

\section{Electrochemical end electron microscopy study}

All voltammetry measurements were carried out with an Ivium Stat.XR electrochemical analyser (Eindoven, Netherlands). A three-electrode cell with gold plate working electrode, having $1.00 \mathrm{~cm} 2$ surface area, platinum wire auxiliary and $\mathrm{Ag} / \mathrm{AgCl}(3$

This work was supported by EU H2020 Marie Sklodowska-Curie Individual Fellowship (Grant no: 706694), MIIC Strategic Postdoc Grant and MIIC Seed Grant at Linkoping University (LiU), Sweden.
$\mathrm{M} \mathrm{KCl}$ ) reference electrodes were used in the voltammetry measurements[14]. Temperature controlled SEM was performed using an LEO 155 Gemini (Zeiss, OR, USA) by attaching a H1002 hot-stage module for heating and water cooling apparatus as described in Figure 3.

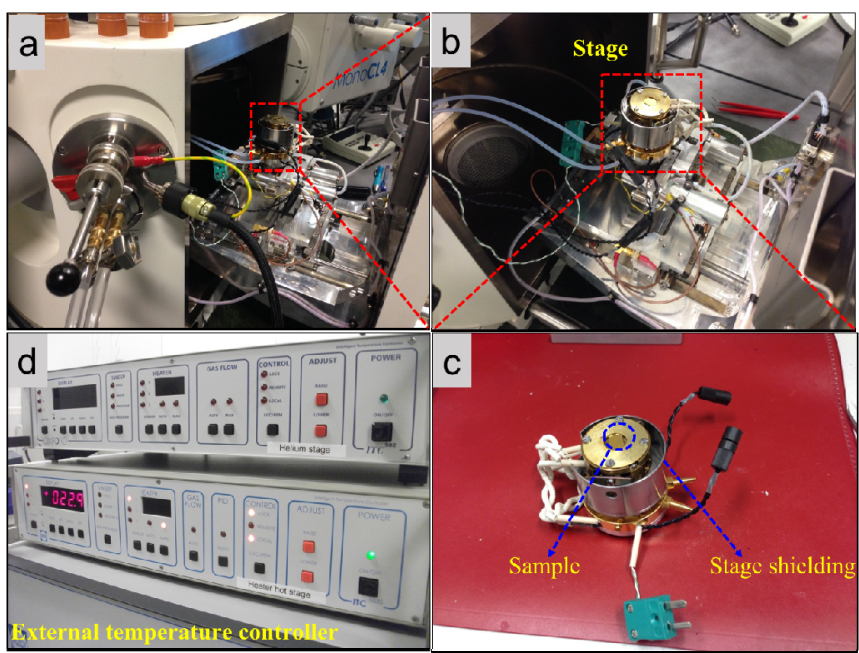

Figure 3: Set-up for temperature-controlled SEM device, stage holder $(a-c)$ and external temperature controller $(d)$.

\section{RESULTS AND DISCUSSION}

In this present report we have developed an aptamer-based platform to detect rare events in real time. Wy-5a is immobilised along with temperature responsive polymer PNIPAAM suitable for the biosystem [15-16].
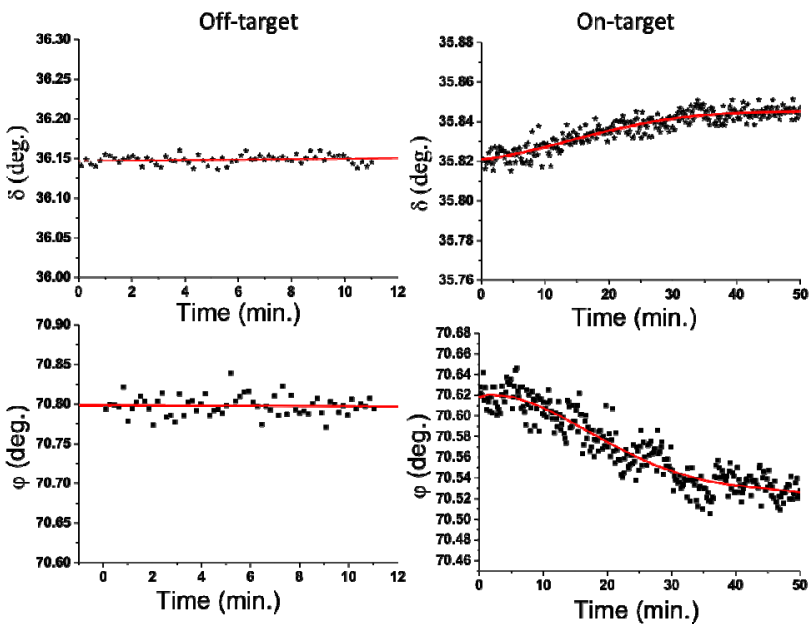

Figure4: In situ real time target cell sensing

The surface of the sensor is being characterised and optimised by VASE and found regions that could be exploited for sensing events independently irrespective of the wavelength. To validate the hypothesis a proof-of-concept set up has been developed where we have used a specifically designed cuvette to measure the response with real-time. It has been found that sensor is not responding when the target cell is absent. PBMC have been used to mimic the similar blood mimicking condition as off-target in Figure 4. However, we have found a consistent change in delta and psi value in presence of the 
specific target cells (PC3) with respect to time at $37^{\circ} \mathrm{C}$.
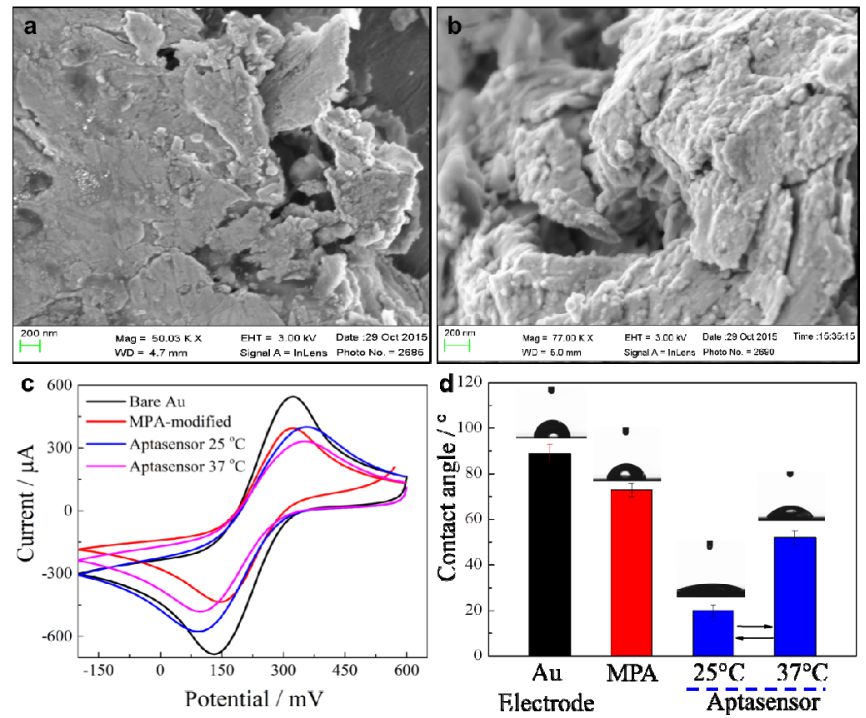

Figure 5: Switchable sensor surface. The electron microscopic structural change induced by the temperature (a to b) and reflected by their electrochemical (c) and contact angle measurement (d)

The polarization change is represented as an amplitude ratio, $\Psi$, and the phase difference, $\Delta$ as described in Figure 4. This confirms the capture of the target cells on the sensor surface as reconfirmed by Laser Scanning Confocal Microscopy (LSCM) as shown in Figure 6 (left panel). We have found that in a blood mimicking condition with similar cell population and flow, we can sense in situ up to 10 target cells $/ \mathrm{ml}$ of blood where the background cell count in $10^{9}$.

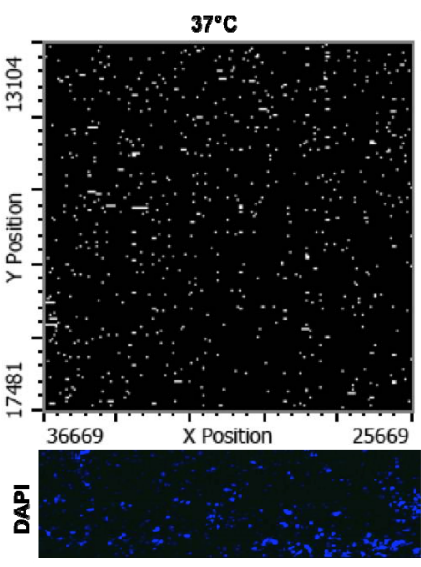

Capture

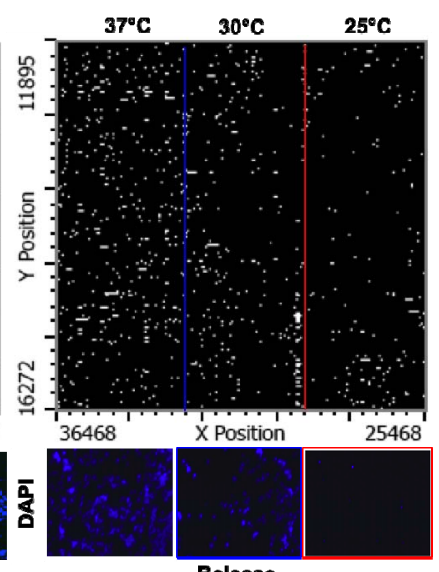

Release
Figure 6: LSCM images of real captured and release cells from the sensor after detection for further advanced diagnosis

The developed sensor surface has controllable thermoswitchable properties as showed in Figure 5. After capturing the target cells, upon reducing the temperature to $25^{\circ} \mathrm{C} \mathrm{()} \mathrm{can}$ allow to remove the cells from the sensor surface as illustrated in Figure 7 and showed directly in LSCM release data in Figure 6 . The safe removal of $95 \%$ live target cells was further reconfirmed by flow cytometry (Figure $7 b$ ) with dual probe Po-pro and 7-AAD. The idea is to recover those captured cells for further advanced diagnosis such as tumour staging etc.

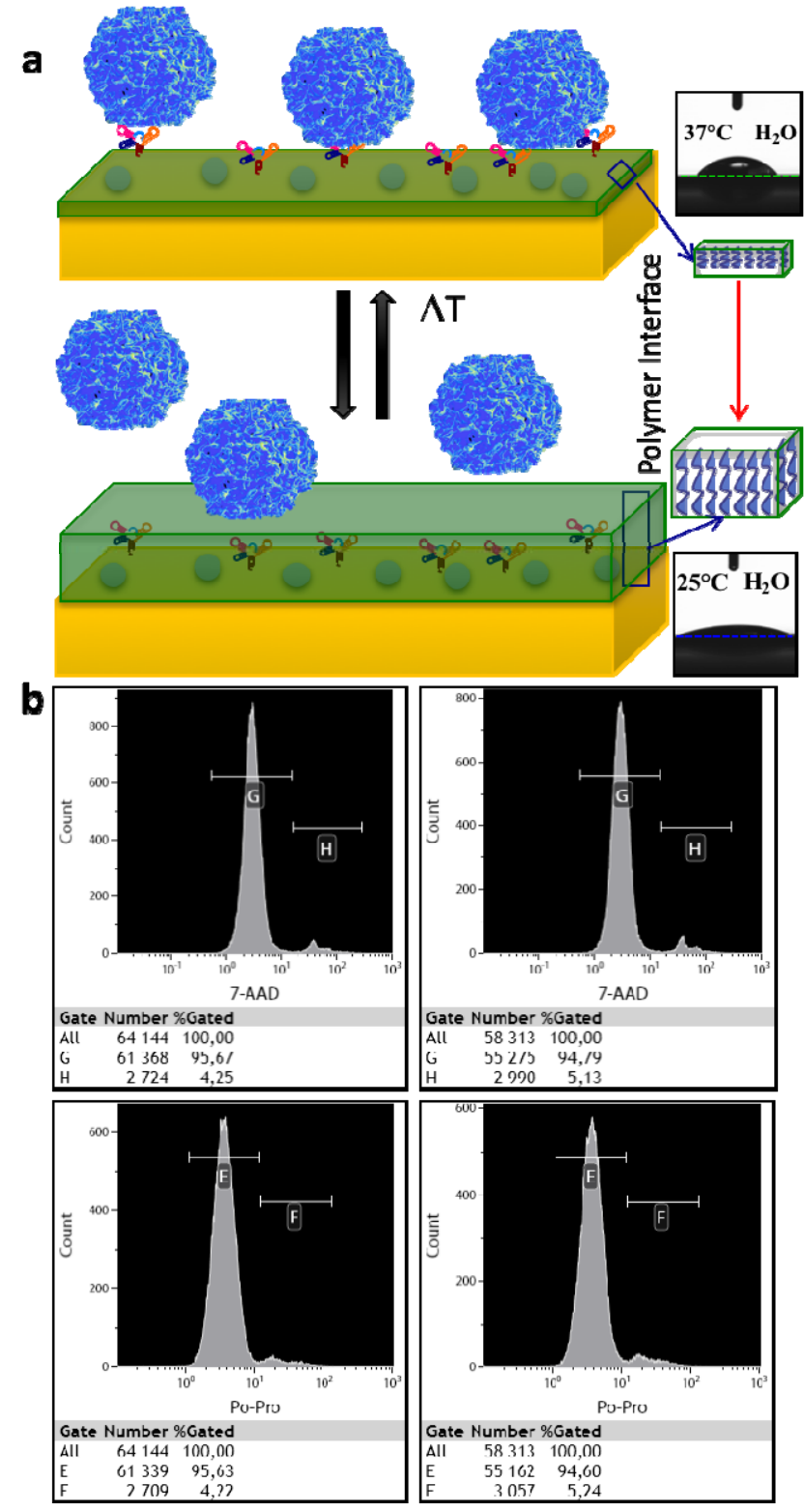

Figure 7: Towards in situ biopsy for detection and capture of cancer cell in its early stage of asymptomatic patients before the onset of metastasis.

\section{CONCLUSION}

An integrated label-free aptasensor reported that is equipped with switchable architecture enable us to detect, capture and release the target cells through a non-enzymatic and mechanothermal procedure with biocompatible temperature range 20 $37^{\circ} \mathrm{C}$ for further advanced clinical analysis.

\section{ACKNOWLEDGMENT}

H.K.P thanks Dr. S. Deb and S. Ramstrom (isolation of PBMC), O. Parlak (electrochemistry and SEM), Dr. J.J. Reddy (Flow cytometry and LSCM) and Dr. S. Valyukh (Ellipsometry) for helping with the experiments at LiU, Sweden. H.K.P. wants to cordially thank Prof. A.P.F Turner's group for helping and hosting this project inside Biosensors and Bioelectronics Centre, LiU, Sweden. 


\section{REFERENCES}

[1] http://www.who.int/news-room/detail/03-02-2017-early-cancerdiagnosis-saves-lives-cuts-treatment-costs

[2] Hazelton, William D., and E. Georg Luebeck. "Biomarker-based early cancer detection: is it achievable?." Science translational medicine 3, no. 109 (2011): 109fs9.

[3] Soman, Chinmay P., and Todd D. Giorgio. "Quantum dot self-assembly for protein detection with sub-picomolar sensitivity." Langmuir 24, no. 8 (2008): 4399-4404.

[4] Felipe De Sousa, E. Melo, Louis Vermeulen, Evelyn Fessler, and Jan Paul Medema. "Cancer heterogeneity — a multifaceted view." EMBO reports 14, no. 8 (2013): 686-695.

[5] Fisher, R., L. Pusztai, and C. Swanton. "Cancer heterogeneity: implications for targeted therapeutics." British journal of cancer 108, no. 3 (2013): 479.

[6] Tang, Dean G. "Understanding cancer stem cell heterogeneity and plasticity." Cell research 22, no. 3 (2012): 457.

[7] Gao, Hui, Joshua M. Korn, Stéphane Ferretti, John E. Monahan, Youzhen Wang, Mallika Singh, Chao Zhang et al. "High-throughput screening using patient-derived tumor xenografts to predict clinical trial drug response." Nature medicine 21, no. 11 (2015): 1318.

[8] Schoukroun-Barnes, Lauren R., Florika C. Macazo, Brenda Gutierrez, Justine Lottermoser, Juan Liu, and Ryan J. White. "Reagentless, structure-switching, electrochemical aptamer-based sensors." Annual Review of Analytical Chemistry 9 (2016): 163-181.

[9] Shangguan, Dihua, Tao Bing, and Nan Zhang. "Cell-SELEX: Aptamer selection against whole cells." In Aptamers Selected by Cell-SELEX for Theranostics, pp. 13-33. Springer, Berlin, Heidelberg, 2015.
[10] Li, Wan-Ming, Tao Bing, Jia-Yi Wei, Zhe-Zhou Chen, Di-Hua Shangguan, and Jin Fang. "Cell-SELEX-based selection of aptamers that recognize distinct targets on metastatic colorectal cancer cells." Biomaterials 35, no. 25 (2014): 6998-7007.

[11] Civit, Laia, Seyed Mohammad Taghdisi, Anna Jonczyk, Silvana K. Haßel, Carsten Gröber, Michael Blank, H. James Stunden et al. "Systematic evaluation of Cell-SELEX enriched aptamers binding to breast cancer cells." Biochimie 145 (2018): 53-62.

[12] Wang, Yuanyuan, Yun Luo, Tao Bing, Zheng Chen, Minhua Lu, Nan Zhang, Dihua Shangguan, and Xin Gao. "DNA aptamer evolved by cellSELEX for recognition of prostate cancer." PLoS One 9, no. 6 (2014): e100243.

[13] Berlind, Torun, Michal Poksinski, Pentti Tengvall, and Hans Arwin. "Formation and cross-linking of fibrinogen layers monitored with in situ spectroscopic ellipsometry." Colloids and Surfaces B: Biointerfaces 75, no. 2 (2010): 410-417.

[14] Parlak, Onur, Anthony PF Turner, and Ashutosh Tiwari. "On/Off-Switchable Zipper-Like Bioelectronics on a Graphene Interface." Advanced Materials 26, no. 3 (2014): 482-486.

[15] Ravichandran, Ranjithkumar, C. Astrand, Hirak Kumar Patra, Anthony PF Turner, Véronique Chotteau, and Jaywant Phopase. "Intelligent ECM mimetic injectable scaffolds based on functional collagen building blocks for tissue engineering and biomedical applications." RSC Advances 7, no. 34 (2017): 21068-21078.

[16] Patra, Hirak K., Yashpal Sharma, Mohammad Mirazul Islam, Mohammad Javad Jafari, N. Arul Murugan, Hisatoshi Kobayashi, Anthony PF Turner, and Ashutosh Tiwari. "Inflammation-sensitive in situ smart scaffolding for regenerative medicine." Nanoscale 8, no. 39 (2016): 17213-17222. 